\title{
The relationship between television advertising, children's viewing and their requests to Father Christmas.
}

With children in the UK watching an average of $2 \frac{1}{2}$ hours of television per day, $63 \%$ possessing a television in their bedroom ${ }^{1}$ and the growth of commercial TV channels in the UK, the potential exposure to television advertising for today's children is considerable. There is now a growing body of literature examining the effects of television advertising on children, their understanding of it and the extent of its influence. This research is highly relevant to those concerned with the regulation of advertising to children and those who see a need to protect children from aggressive marketing ${ }^{2}$. Young children are a vulnerable group, it is suggested, because they lack the cognitive skills necessary to defend themselves against the persuasive power of advertising ${ }^{3}$. This paper takes account of theories about children's development in assessing this vulnerability and uses a methodology that examines children's behavioural responses to television advertising.

Advertisers are not completely free to bombard children with television advertising as regulations control the amount and type of television advertising targeted at children in many parts of Europe and the US. Nevertheless, controls vary considerably from country to country ranging from few restrictions to timing restrictions to total bans on any TV advertising which is aimed directly at children ${ }^{4}$. In the UK the content and amount of advertising are regulated ${ }^{5}$. Nonetheless, whilst strict regulations are in place, there remains plenty of opportunity for advertisers to target children, leading to claims of increasing commercial pressure on children ${ }^{6}$.

Research suggests that young children lack an explicit understanding of the advocatory nature of advertising and that this renders them more susceptible to its effects. Oates, Blades \& Gunter ${ }^{7}$ suggest that young children may not understand the 
link between advertising and commercial profit. This supports previous research which found that only $25 \%$ of $11-12$ year olds were able to explain advertising motives in terms of selling and profit ${ }^{8}$, and children only appear to begin to understand the persuasive intent of an advertisement at around 7-8 years old ${ }^{9}$. Before this age, children see the purpose of adverts as helping, entertaining or informing viewers ${ }^{10}$.

Such understanding is important because, although adults recognise that an advertisement may present a biased message designed to sell something, young children are unaware of this. Children are also less likely to have experienced disappointment with advertised products they have bought and are less able to delay gratification ${ }^{11}$. Furthermore, children may be more susceptible to peripheral routes of persuasion (e.g. celebrity endorsement or cartoon presentation) ${ }^{12,13}$ even though some argue that young children's limited memory capacity means they will not recall advertisements ${ }^{14}$.

Critical to the issue of assessing the true impact of advertisements on children is employment of an age-appropriate methodology. To this end, our earlier research examined children's letters to Father Christmas to investigate the impact of toy advertising at Christmas on 3 to 6 year olds. ${ }^{10}$. Children in the UK who watched more commercial television requested significantly more items. However, poor brand recall was noticeable and, excepting requests for Barbie and Action Man, there was little relationship between the products advertised and the children's brand-named requests. The UK children were also compared with a similar group in Sweden, where childtarget advertising is banned, and were found to request significantly more items. Again this suggests that advertising was exerting an influence, even though brand recall was poor. A subsequent study with 4-5 year olds suggests that this failure to request brandnamed advertised products is attributable more to differences between recall and recognition memory in children of this age, than with advertising ${ }^{15}$. The young 
children in this study were shown to have high recognition for advertised brands and this effect was particularly notable for girls, which may be due to early socialisation processes or the fact that better verbal skills at this age facilitates their processing of the messages in advertisements.

This discrepancy between high brand recognition and poor recall is explained by the way children's conceptual knowledge develops. According to Karmiloff-Smith's Representational Redescription (RR) model ${ }^{16}$, knowledge is initially implicit and nonverbal and can only be accessed via recognition tasks. This knowledge, over time, becomes explicit and verbally accessible until, at a later stage, children can remember adverts and explicitly recall brand names. This same model can also explain other anomalies found in advertising research with children. For example, in contrast with the studies mentioned earlier which demonstrate explicit knowledge not emerging until around the age of eight, Pine \& Veasey found that children as young as four years old have an implicit understanding of the positive bias in promotional messages ${ }^{17}$.

The study reported here replicates the original letters to Father Christmas paradigm, but with children aged six to eight years old ${ }^{10}$. This methodology has high ecological validity, as it measures a natural activity that children engage in, i.e. writing letters to Father Christmas, and uses a natural time frame between exposure to toy manufacturers' advertising and writing the letters. Poor brand recall in the earlier study was attributed to the children being too young to explicitly recall the names of the toys they had seen, even though exposure to television had increased their desire. The children in this study are older (6-8 years) and, if they have more explicit knowledge, will be more likely to request items by brand name thus enabling firmer conclusions to be drawn about the impact of television advertising. 
Our main prediction was that children who watch more television would request more advertised toys, by brand-name, in their letters to Father Christmas. Furthermore we expected this to be greater for children who favour watching commercial television channels over non-commercial channels. We were also interested in whether there would be gender differences, as findings from earlier studies have suggested that girls may be more influenced by advertising than boys ${ }^{15}$. It was also predicted that there would be a relationship between the frequency with which products are advertised and the number of requests for these products.

\section{METHOD}

Design

A correlational design examined the relationship between the amount of television watched and the proportion of advertised items requested by children in letters to Santa. In a between-groups design, a 3 (viewing category) x 2 (viewer type) $\mathrm{x}$ 2 (gender) design analysed the proportion of advertised item requests. Viewing category had three levels (low, medium and high frequency) whilst viewer type had two (commercial and non-commercial channels).

A second correlational analysis examined the relationship between children's requests and the products advertised during children's TV programmes during the six week period before the children wrote their letters. The principle variables were the frequency with which the products were advertised, and the frequency with which those items were requested.

Participants 
Children were recruited from Year 2 of an infants' school and Year 3 of a junior school, both in Hertfordshire, UK. The schools were based in areas of mixed housing from households with varied income. From 111 letters seeking participation permission for the children from their parents, consent was refused for only one child. Thus, 110 children were interviewed initially. However, complete data (interview and letter) was collated on only 98 children.

The sample comprised 52 boys and 46 girls, with a mean age of 7.3 years (SD = 6.8 months). There were 50 children in Year 2 ( 28 boys and 22 girls), with a mean age of 6.10 ( $\mathrm{SD}=3.3$ months $)$ and an age range from 6 years 4 months to 7 years 3 months. Forty-eight children from Year 3 took part (24 boys and 24 girls), with a mean age of $7.9(\mathrm{SD}=3.7$ months $)$ and ages ranging from 7 years 4 months to 8 years 3 months.

\section{Materials}

A questionnaire was used in an interview situation to gather data on children's viewing habits. Adapted from the Television Viewing Habits Questionnaire ${ }^{10}$, this instrument assessed relative frequency of viewing, rather than the number of hours of television watched, which is difficult to accurately assess with children. An overall TV viewing score was derived from four questions, such as "How often do you watch television in the evening during the week, Monday to Friday?" Multiple choice response options were provided for the children. A response of "Every day" scored 3 points, "lots of days" scored 2 points, "just some days" scored 1 point and "never" was scored as 0 . The other three questions were related to television viewing in the morning on weekdays, Saturdays and Sundays and were scored in a similar way. Greater frequency of viewing therefore equals a higher TV viewing score and provides a continuous score. However, because it is relative in nature, the score lends itself to 
categorising the children as low, medium and high viewers, based on the equal division of the maximum possible score of 12. Thus, a child with a score between one and four was classified as a low viewer; between five and eight as a medium viewer; and between nine and twelve as a high viewer.

The questionnaire also included a measure of preference with regard to commercial and non-commercial viewing. Commercial television channels screen paidfor advertising for products and services, whilst non-commercial channels do not carry advertising at all (e.g. the BBC in the UK). By asking children which programmes they liked to watch on weekday and weekend mornings and evenings, the child's preference for commercial or non-commercial channels could be determined. If, on all four occasions, a child indicated only programmes on non-commercial channels they were classified as non-commercial viewers. If any, or all, of the programmes indicated were on commercial channels they were classified as commercial viewers. This measure indicated a preference rather than constituting a quantitative measure. Nonetheless children who preferred watching non-commercial channels were considered less likely to be exposed to advertising.

Children were also asked whether they knew what a television advertisement is, with yes, no and not sure options as responses. A further, open-ended, question gave them the opportunity to answer freely.

Interviews with the children were taped using a Sony TCM939 cassette recorder. Video recordings were made of a selection of children's programmes on terrestrial (nonsubscription) and satellite/cable (subscription) commercial television channels between Saturday 29 October 2005 and Saturday 10 December 2005 in order to determine the advertising shown. A total of 76 hours of children's commercial television programmes were recorded, comprising 38 hours of UK non-subscription television programmes 
from ITV and Channel 5, and 38 hours of subscription programming on Nickelodeon, Boomerang, Cartoon Network, Nick Junior, and Jettix. The video recordings were taped using a Sanyo HVR-777E video recorder. Details of the advertisements shown were noted by the experimenter who recorded the type of product and brand name. Advertisements were also identified as being targeted at girls, boys or both. Only clear gender differences were noted, e.g. Barbie for girls, Hotwheels for boys, Cluedo for both.

Letters to Father Christmas were written on paper provided by the schools. A count was made of the items requested in the letter and these were categorised as nonbranded, or generic products (e.g. doll, or puppy), as branded, advertised items (such as Hot Wheels cars, or Bratz dolls), and branded, but not advertised, items (such as Yu-GiOh! cards and Tamagotchis). The analyses in the results section was carried out on only the branded, advertised items recorded in the previous six weeks.

Procedure

The children wrote their letters in class during the week commencing 12th December. In order to keep the study naturalistic, the request to the children to write letters to Father Christmas came from their teachers and the researcher was not present.

Interviews using the questionnaire were conducted between 5 and 14 December. Each child was seen alone in a quiet room. After a brief explanation about the purpose of the study, the interview took place. At the end of the interview, each child was thanked for their assistance.

\section{RESULTS}


Analysis of questionnaire data

TV Viewing Scores. Children's television viewing habits were compiled into a score based on their responses to the questionnaire items. A child's raw television score was therefore between $0-12$. The mean television score was $\mathrm{M}=7.51(\mathrm{SD}=2.6)$. Scores ranged from 1 to 12 with a slightly negatively skewed distribution (-.27) and no outliers. The mode television score was 7 . Thus, both the mean and the mode scores were within the 'medium viewer' category. A one-way ANOVA was conducted to determine whether there was any difference in the amount of television watched by girls $(\mathrm{M}=7.57, \mathrm{SD}=2.47)$ and boys $(\mathrm{M}=7.46, \mathrm{SD}=2.73)$. There was no significant effect of gender on TV score, $\mathrm{F}(1,96)=.04, \mathrm{p}=.85$.

These scores were then categorised into low (1-4), medium (5-8) and high scores (9-12). This variable, relating to the amount of television viewing, is referred to in the results as 'viewing category'.

'Viewer type' was based on preference for viewing programmes on commercial or non-commercial television. Fifty six children were categorised as commercial television viewers and 42 were categorised as non-commercial viewers. Table 1 indicates how viewing types were distributed by viewer category.

\section{(Table 1 about here)}

The association between viewer type (commercial or non-commercial) and viewing category (low, medium or high) was assessed with a chi square test. This was found to be significant, $\chi^{2}(\mathrm{df}=2), \mathrm{n}=98=6.095, \mathrm{p}=0.047$, and examination of the frequencies suggests that children whose viewing frequency was 'high' were more likely to prefer watching commercial channels. 


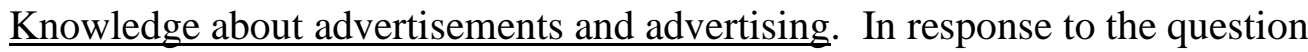
about advertising 51 children (52\%) replied that they knew what an advertisement was, and were able to give some kind of explanation. Another 14 children (14\%) were not sure, and 33 (34\%) said they did not know.

The second part of this question invited the child to tell the experimenter about advertisements. In order to show understanding of the difference between an advertisement and a programme, a child needed to either give an example of an advert they had seen, or to give a general explanation about adverts. Some children responded that they did know what an advert is, but gave erroneous explanations, such as "they show you what programme is on next". Such a response led to the child being coded as not understanding the purpose of advertisements. However, more typical explanations related to the informative nature of advertisements, such as, "they tell you if there is something new in the shops", or "they tell you what you can buy". If children were able to demonstrate general knowledge of advertising of this nature they were classified as 'having some knowledge' of advertising, albeit not complete. However, only one child from the original sample of 106 included an explanation of the advertiser's intent as she stated that, "It's about what people want you to get. It tells you where to buy toys and things". A chi-square test was performed to look for an association between the viewer type (commercial or non-commercial) and knowledge about advertisements. This was not significant, $\chi^{2}(\mathrm{df}=2)=.998, \mathrm{p}=>0.05$.

TV Viewing and Requests Made to Father Christmas.

$\underline{\text { Requests. }}$ Each letter was analysed for a count of the number of items the child had requested. The number of total items requested per child ranged from 1-18 with a 
mean of $4.57(\mathrm{SD}=3.45)$ and a mode of 5. Advertised item requests ranged from $0-8$, with a mean of $2.02(\mathrm{SD}=1.98)$.

As the number of requests made by each child varied considerably, further calculations were carried out based on the proportion of advertised products requested by each child in relation to the total number of requests in the letter. This was calculated by dividing the number of requested advertised items by the total number of requests made, and expressed as a percentage. The mean proportional brand request per letter was $46.05 \%(\mathrm{SD}=36.47 \%)$.

The Relationship between Requests for Advertised Items and Television Score. The relationship between television viewing score and the proportion of requests for advertised items was investigated using a one-tailed Pearson correlation coefficient. There was a significant, positive correlation between the two variables, $r(98)=0.27, p=$ 0.004. Although this is a weak correlation, it nonetheless suggests that proportionally greater numbers of requests for advertised, brand-named items were associated with higher television scores.

$\underline{\text { Requests for advertised items by Viewer Type, Gender and Viewing Category. }}$ A three-way ANOVA was performed to determine whether the independent variables of viewer type (commercial or non-commercial), gender (male or female) or viewing category (low, medium, high) influenced advertised brand requests.

Commercial viewers' letters contained a larger proportion of advertised items $(\mathrm{M}=56.55, \mathrm{SD}=35.73)$ than non-commercial viewers $(\mathrm{M}=32.05, \mathrm{SD}=32.91)$, and the main effect of viewer type was significant, $F(1,86)=10.17, p=.002$, partial $\eta^{2}=$ .11 .

There was a main effect of gender on advertised brand requests as a proportion of total requests, $\mathrm{F}(1,86)=8.52, \mathrm{p}=0.004$, partial $\left.\eta^{2}=.205\right)$ and from analysis of the 
means, it would appear that girls requested proportionally more advertised items $(\mathrm{M}=$ $56.13, \mathrm{SD}=35.47)$ than boys $(\mathrm{M}=37.13, \mathrm{SD}=35.32)$.

There was also a main effect of viewing category on the proportion of advertised products requested, $F(2,86)=8.59, p=.000$, partial $\eta^{2}=.17$. Post hoc Bonferroni tests showed that the letters of children categorized as low viewers contained a lower proportion of advertised items $(\mathrm{M}=8.54, \mathrm{SD}=13.71)$ than either medium $(\mathrm{M}=47.31$, $\mathrm{SD}=34.75)$ or high viewers $(\mathrm{M}=57.59, \mathrm{SD}=36.02)$. There were no interaction effects.

Relationship between television advertisements and brand items requested The 76 hours of children's programmes recorded contained a total of 2,879 advertisements. Of these 5.4\% were for 'other' items, 7.7\% were for food items and $87 \%$, or 2523 advertisements, were for toys (more than 33 advertisements per hour). In addition to the usual type of toys such as cars, dolls and games, the category 'toys' included advertisements for DVDs or videos aimed at children e.g. 'Madagascar' or 'Pingu', plus music CDs such as 'Disney's Greatest Hits', and 'Pop Party 3'. CDs for specific bands or groups were not included. Overall, the toys category comprised 306 separate products, which were collapsed into 231 brands (e.g. Bratz, Gr8 Gear, Disney).

Of the recorded advertisements, 83 products appeared to be aimed at boys only (e.g. Hot Wheels), 88 were aimed at girls only (e.g. Barbie), and 135 could be classified as non-gender specific (e.g. Cluedo). However, although the overall numbers of products aimed specifically at boys or girls appeared similar, there were large differences in the frequency with which these products were advertised, resulting in far higher targeting of girls than boys. Of the 2523 advertisements, 645 (25.6\%) were 
targeted at boys, whilst almost half as many again (942 or $37.3 \%$ ) were targeted at girls. A further $936(37 \%)$ were non-gender specific.

Out of a total of 448 requests made by the children, 198 (44\%) were for branded, advertised items. The ten most heavily advertised brands account for $47.9 \%$ of advertised products requested, see Table 2 .

\section{(Table 2 about here)}

The relationship between the most heavily advertised items (number of times a brand was advertised) and the requests (number of requests) made by children was investigated using Pearson product-moment correlation coefficient. There was a positive correlation, approaching significance, $\mathrm{r}(10)=0.63, \mathrm{p}=0.053$, suggesting that children's requests may be associated with heavily advertised items.

As Table 2 indicates, the most requested brand was for Bratz doll products. Advertising of this brand accounted for $6.1 \%$ of toy advertisements recorded on terrestrial, and $5.3 \%$ of toy advertisements advertised on non-terrestrial television. This was only surpassed by the Barbie brand which accounted for $9.6 \%$ of toy advertisements on terrestrial television and $8.5 \%$ of toy advertisements on nonterrestrial television. Barbie products were requested by 19 children (or almost onefifth of the sample).

Six out of the ten most heavily advertised brands were aimed at girls. Girls made a total of 253 requests, of which 130 were brand items. Requests for these six brands accounted for $60.7 \%$ of girls' advertised brand requests, or $31 \%$ of their total requests.

Boys made a total of 195 requests, of which 68 (35\%) were for advertised brand items. Of the brand requests, $22 \%$ were for the four brands in the ten most heavily advertised brands aimed expressly at boys. 
Non-Advertised Brand Requests._A total of 73 requests were made for products that were brand named but not advertised. This represents $16 \%$ of the total requests. This data has been excluded from further statistical analysis, but is given consideration in the discussion.

\section{DISCUSSION}

This study examined the relationship between television advertising and the advertised products requested by children aged 6 to 8 years old in their letters to Father Christmas. Based on self-report data, with no parental validation, a questionnaire took account of television viewing habits, the viewer type (whether children preferred watching commercial or non-commercial television) and gender. Finally, it examined the relationship between advertising shown on children's television in the pre-Christmas period and children's requests. The research was a replication of a previous study ${ }^{10}$ but with older children to examine whether they would include more brand-named items in their letters to Father Christmas as a result of better recall.

The findings confirmed that greater exposure to television led to an increase in the proportion of advertised products requested. Furthermore, children who favoured watching commercial television were likely to request more advertised products than those children who said they preferred watching non-commercial television. The gender effects found in previous research were replicated ${ }^{15}$, with girls requesting more advertised products than boys. There was also a strong relationship between the most heavily advertised products and the most requested products, despite a few of the most heavily advertised products not being requested at all.

Developmental psychologists have been concerned with elucidating children's understanding of advertising, particularly in relation to theories about explicit 
knowledge and cognitive defences. More children in this study (52\%) showed explicit understanding of what an advertisement is than the younger children in the Pine \& Nash ${ }^{10}$ study (32\%), suggesting age-related increases in knowledge. However, their explanations still indicated that they saw advertising as informative rather than persuasive, with only one child demonstrating understanding of the motive of the advertiser. Furthermore, viewers who showed a preference for commercial television were not better informed. Thus, favouring commercial television channels does not alone appear to facilitate the development of the cognitive skills necessary to aid critical understanding. This suggests that whilst age may confer benefits in terms of knowledge development, the findings relate particularly well to the idea of this gradually developing from an implicit understanding through more explicit phases ${ }^{16}$. This would suggest that some of these children are able to abstract a certain amount of knowledge from their implicit understanding of advertising, but that this knowledge is not complete.

The six to eight-year-old children in this study asked for more products by brand name than the younger children in the earlier study ${ }^{10}$ which may be attributable to their increased memory capacity and explicit knowledge. Therefore, rather than consider the development of cognitive defences against advertising at this age, as suggested by some, this study demonstrates that it is perhaps more pertinent to consider whether knowledge about advertising in its early phases of development combined with better recall for brand names actually leaves children more vulnerable around this particular age. Moreover, it raises the question of whether advertising directed at children is fair, especially in view of the number of advertisements being shown - as many as 51 in one hour in this study - and whether, as a result, vulnerability is paramount at this age. 
Aside from the non-branded requests, two other categories of request merit some consideration insofar as they shed light on children's consumer behaviour. One is where branded goods have been requested, but not advertised and the other is where goods have been advertised, but not requested. In this study requests were made for Harry Potter items (e.g. books, figures, etc.), Playstation 2s and Gameboys and yet these were not specifically advertised. However, these requests may have arisen indirectly from advertising as games for the games consoles were advertised and the latest Harry Potter film was heavily promoted at the time. Yu-Gi-Oh cards, requested by eight children, may also have benefited from indirect advertising. These appear to be similar to the Pokemon phenomenon, in that the $\mathrm{Yu}-\mathrm{Gi}-\mathrm{Oh}$ programme is in itself an advertisement for the product. It seems that even in the absence of specific advertisements, advertising may still account for a number of requests. Whilst word-ofmouth and peer pressure have been indicated as influences on purchases ${ }^{18}$ it seems that only Tamagotchis benefited in this way in this study with fifteen requests being made for these items.

However, whilst advertising clearly carried influence, the children in the present study only seemed to be affected when the advertisements carry sufficient appeal. For example, of the 231 brands advertised, 182 (79\%) were not requested at all. This could have occurred for a number of reasons. Firstly, some items were unlikely to appeal to this age group (e.g. Postman Pat, Bob the Builder). Secondly, children may already possess a number of the products advertised. Thirdly, they may have critically evaluated the products and decided that some items, or perhaps advertisements, were just not appealing enough.

In terms of gender effects, the findings both in this study and in previous research ${ }^{10,15}$ suggest that girls are more susceptible to advertising and request more 
branded products. For example, one seven-year old girl's letter specifically stated that she wanted a toy that she had seen on TV and recounted the retail outlets that sold it. She went on to say, 'I want it because it looks good fun and if one of your friends has a pixel chick they can hang out together and play with each other'. She had clearly assimilated the benefit that the manufacturer of the toy set out to convey in their advertisement, and even used the same terminology, "hang out", that was used in the advertisement. However, the higher number of branded requests by girls in this study may be also attributable to more advertising appearing to be targeted at girls than boys. This implies that girls are more actively encouraged to become consumers from an early age and accords with findings ${ }^{15}$ that girls recognise brand names at an earlier age to boys.

More generally, the practical implications of children as young as six being targeted as consumers before they have acquired understanding of the true nature of consumer practice (brand development, marketing, sales and profit etc.) are of concern. These young children will not be able to counter the messages they see with the healthy degree of scepticism possessed by adults.

The findings in this study confirm not only that television advertising aimed at children works, but that it works particularly well with children aged six to eight, and it is likely that this can be attributed specifically to children's development and their level of understanding of advertising. In an age when Government is keen to promote sex and drug education in primary schools, this suggests that there is also a need for commercial and critical awareness of advertising on the curriculum. However this must be at a level suitable for this age group and presented so as to be effective in reducing the persuasive effects of advertising, and not simply be informative ${ }^{13,19,20}$. 
Television advertising and children 17

Word count: 4618 


\section{References}

$1 \quad$ Livingstone S, Bovill D. Social perspectives: Have the media ruined childhood? Interactions 1999; 6: 36-41.

2 American Academy of Pediatricians, Committee on Communications. Children, adolescents and advertising. Pediatrics 2006; 118: 2563-2569 [published correction appears in Pediatrics. 2007;119:424]

3 Strasburger VC. Children and TV advertising: Nowhere to run, nowhere to hide. J Dev Behav Pediatr 2001; 22(3): 185-187.

4 Buijzen M, Valkenburg PM. The effects of television advertising on materialism, parent-child conflict, and unhappiness: A review of research. $J$ Appl Dev Psychol 2003; 24: 437-56.

5 BCAP. Television Advertising Standards Code. In, Vol. 2005: Broadcast Committee of Advertising Practice, 2004.

6 Valkenburg PM. Media and youth consumerism. J Adolesc Health 2000; 27S: $52-6$

7 Oates C, Blades M, Gunter B. Children and television advertising: When do they understand persuasive intent? J Consum Behav 2002; 1: 238-45.

8 Ward S, Wackman DB, Wartella E. How children learn to buy: the development of consumer information-processing skills. Beverly Hills, CA: Sage, 1977.

9 Chan K. Hong Kong children's understanding of television advertising. J Market Commun 2000; 6: 37-52.

10 Pine KJ, Nash A. Dear Santa: The effects of television advertising on young children. Int J Behav Dev 2002; 26: 529-39.

11 Kopp CB. The growth of self-regulation: Caregivers and children. In: Contemporary topics in developmental psychology (Eisenberg N, ed). New York: Wiley, 1987.

12 Nash AS. Understanding children's thinking about alcohol advertisements on television: a cognitive developmental approach. In: School of Psychology. Hatfield: University of Hertfordshire, 2005.

13 Livingstone S, Helsper EJ. Does advertising literacy mediate the effects of advertising on children? A critical examination of two linked research literatures in relation to obesity and food choice. J Commun 2006; 56: 560-84.

14 Macklin MC. The effects of an advertising retrieval cue on young children's memory and brand evaluations. Psychol Market 1994; 11: 291-311.

15 Pine KJ, Nash A. Barbie or Betty? Brand awareness in pre-school children and evidence for gender linked differences. J Dev Behav Pediatr 2003; 24. 
16 Karmiloff Smith A. Beyond modularity: A developmental perspective on cognitive science. New York: OUP, 1992.

17 Pine KJ, Veasey T. Conceptualising and assessing young children's knowledge of television advertising within a framework of implicit and explicit knowledge. J Market Manage 2003; 19: 459-73.

18 Achenreiner GB. Materialistic values and susceptibility to influence in children. In: Adv Consum Res (Brucks M, MacInnis DJ, eds), Vol. 24. Provo, UT: Association for Consumer Research, 1997: 82-8.

19 John DR. Consumer socialization of children: A retrospective look at twentyfive years of research. J Consum Res 1999; 26: 183-213.

20 Buckingham D. The media literacy of children and young people: a review of the research literature on behalf of Ofcom. In. London: Ofcom, 2006. 